\title{
Low-mortality death in hospitals after vertical integration with Health Maintenance Organizations
}

Aaron F. Miller, Ashley Hodgson*

Department of Economics, St. Olaf College, United States

Received: April 7, 2017

DOI: $10.5430 /$ jha.v6n4p31
Accepted: June 18, 2017

Online Published: June 27, 2017

URL: https://doi.org/10.5430/jha.v6n4p31

\begin{abstract}
Objective: Hospital mergers and acquisitions continue to rise due to government and market pressures to join Accountable Care Organizations in order to lower costs. Our study examines the changes in a quality of care measure when hospitals were acquired by Health Maintenance Organizations (HMOs) to analyze the effect that vertical integration has on coordination of care.

Methods: Using California patient discharge data from 2000-2011, our analysis used differences-in-differences and logistic models to test for a change in a quality of care measure before and after hospitals merged with an HMO. We utilized Patient Safety Indicator \#2, death rate in low-mortality diagnosis related groups, to measure quality of care.

Results: Hospitals experienced decreases in low-mortality death rates after being acquired by an HMO. This group of hospitals had increasing measures of the quality indicator prior to the merge as well, suggesting selection of well-performing hospitals by HMOs. Hospitals acquired by HMOs also faced increased Type- 2 diabetes rates post-merge.

Conclusions: Our results suggest that hospitals merging with a vertically integrated health care system may lead to increases in quality of care. It appears that this could be due to either HMOs providing more coordinated care, or that HMOs acquire hospitals already trending towards better care.
\end{abstract}

Key Words: Vertical integration, Health Maintenance Organization, Quality of care, Hospital consolidation

\section{INTRODUCTION}

United States hospital consolidations doubled from 2009 to 2012, topping 100 total deals. ${ }^{[1]}$ The majority of such acquisitions have been hospital-to-hospital mergers, or horizontal integrations. However, a growing subset of the consolidations is mergers of hospitals with health systems that provide their own health plans, or vertical integration with HMOs. ${ }^{[1]}$ Over $25 \%$ of all Americans receive their health insurance from Health Maintenance Organizations (HMOs) and enrollment in HMO plans increased by $11 \%$ between 2013 and 2015. ${ }^{[2]}$

Increases in hospital acquisitions are consistent with federal healthcare reforms that incentivize Accountable Care Organizations. It is economically favorable for large health systems to acquire hospitals in an effort to lower costs and better coordinate care. ${ }^{[3]}$ Despite such economic advantages, do hospital mergers with HMOs translate to improved quality of care for patients?

To test for the effect of vertical integration on hospital patient outcomes, we look at the low-mortality death rate at hospitals before and after a merge with an HMO, adjusting for patient demographic and health factors. Our analysis employed inpatient hospital discharge data from California's Office of Statewide Health Planning and Development (OSHPD) to run logistic regressions on 47,442,140 patients discharged

*Correspondence: Ashley Hodgson; Email: hodgsona@ stolaf.edu; Address: Department of Economics, St. Olaf College, United States. 
from California hospitals between 2000 and 2011.

\section{Conceptual framework}

Vertical consolidations are often thought to be a much better option for payers and providers, because one company owns several aspects of the supply chain. Economic theory proposes that vertically integrated healthcare systems should display multiple internal efficiency gains. These efficiencies include: economies of scope, lower transaction costs associated with outsourcing, and incentive to invest in health maintenance efforts which reduce costs in the long run. The financial efficiencies that hospitals glean when consolidating free up resources to devote to improved quality of care. Hospitals have low operating margins despite wide variation in reimbursement rates, and there is low variation in operating margin across hospitals. Together, these facts hint that hospitals likely find places to spend additional profits, and it is reasonable to believe that an investment in quality improvement will rank at the top of the list for discretionary spending.

Economies of scope are cost advantages that result when a firm provides a variety of products, rather than specializing in the production of just one. Economic theory suggests that unit costs decrease as output increases. These cost improvements include productivity gains and waste reduction from the ability to eliminate extraneous costs by operating multiple businesses under a centralized management. In 2014 , the operating margin for an average hospital was $8.3 \%,{ }^{[1]}$ whereas operating margins for five of the largest health insurers was $4.3 \%$ (calculated the mean operating margin using 2014 annual reports from the five largest insurance companies in terms of revenue) ${ }^{[4-8]}$ In both healthcare industries, administrative costs constitute a substantial portion of the expenses, reaching over $25 \%$ in hospitals. ${ }^{[9]}$ As such, vertically integrated health care systems are able to increase economies of scope and operate under a centralized management thereby cutting total administrative costs for both businesses by eliminating duplication. The capability for vertically integrated health care systems to have more available services could lead to increases in coordination of care and cost reductions, both benefitting the consumer and the business.

Vertically integrated firms are expected to have lower transaction costs associated with outsourcing. Transaction costs are the expenses that incur in the purchase of goods and services. In the 1930's, Ronald Coase developed transaction cost economics, which thoroughly underlines the numerous inefficiencies that occur in the existence of transactions. These transactional inefficiencies include arguments that contracts include substantial transaction frictions, such as: interest rates, commission fees, and opportunity costs. ${ }^{[10]}$ In the 1970's, Oliver Williamson expanded on Coase's theory and developed transaction cost theory of integration. Williamson argues that integration can be an effective response when transactional frictions are present. ${ }^{[11]}$ Currently, the healthcare system is filled with transaction frictions due to its fragmented nature between insurance companies, hospitals, physicians and patients. As such, vertical integration addresses these difficulties by consolidating production within a single organization, thereby eliminating the need for contracting among separate firms. ${ }^{[11]}$ Therefore, all production is managed internally by one healthcare system and such economic efficiency gains may get passed down to the consumer.

Incentive for HMO to invest in preventative care to obtain cost-savings; vertically integrated healthcare systems manage the insurance rates for its customers. As such, firms seek healthier patient populations in order to have competitive health insurance prices. Thus, firms have economic incentive to perform care in a way that makes the patient as healthy as possible, in order to obtain cost-savings in the future. The National Commission on Prevention Priorities (NCPP) and the National Business Group on Health (NGBH) found multiple preventative interventions to be cost saving. Among these interventions included: childhood immunization, adult Influenza immunization, counseling on use of low-dose aspirin, and screening and counseling for alcohol and tobacco. ${ }^{[12]}$ Therefore, healthcare systems have incentive to invest in short run preventative care efforts to experience long run cost savings. As a result, patients experience an emphasis on preventative care, which lead to better quality outcomes in the long run. ${ }^{[13]}$ In contrary, horizontally integrated healthcare systems bill directly to private insurance companies not affiliated with their practice. As a result, horizontally integrated healthcare systems may have economic incentive to perform unnecessary diagnostic tests and increase patient admissions to drive up their reimbursement. Patients are more likely to pay higher medical bills and suffer from less focused care because of horizontal integration.

There are generally two sizeable obstacles for vertical partnerships to overcome, which contribute to higher prices and lower quality of care for the patient. First, high acquisition costs for two large entities bear a substantial amount of risk leading to an ability to raise prices and increase admission rates. Second, consolidation could lead to monopoly of the market, subsequently leading to higher prices through reduced competition.

Vertical mergers of all sorts assume expensive acquisition costs. For example, in 2015 Kaiser Permanente had an operating income of $\$ 1.9$ billion, compared to Seattle-based 
Group Health Cooperative's $\$ 740$ million. However, Kaiser Permanente paid a total of $\$ 1.8$ billion in 2015 to acquire Group Health Cooperative to expand its geographic reach. Kaiser Permanente is bearing a substantial amount of risk when putting forth costs over two times greater than the operating income of Group Health Cooperative the preceding year, without a guaranteed return on investment. As a result, economic pressures for Kaiser Permanente to recoup its investment could lead to increases in price, admissions, and diagnostic testing. These in turn could lead to higher prices for consumers and possibly less focused care.

Monopoly of the market; antitrust laws regulate the conduct and organization of business corporations to promote fair competition for the benefit of consumers. These laws exist to contain costs, improve quality, expand choice, and incentivize innovation. ${ }^{[14]}$ All companies must abide by these antitrust laws before proceeding through any consolidation to ensure that it will not harm the consumer. However, in 2015 the Federal Trade Commission narrowed its definition of what constitutes an anti-competitive merger. ${ }^{[15]}$ This will likely encourage more large, vertical consolidations in the healthcare industry. This raises concerns for consumers because as healthcare systems increase market share in geographical locations with little competition, firms can drive up prices for an inelastic good like healthcare.

In total, this paper intends to empirically investigate whether such operational efficiencies lead to improved health outcomes for patients.

\section{METHODOLOGY}

\subsection{Data and group selection process}

In order to examine quality of care in hospitals affiliated with HMOs versus those that are not, we used a differencesin-differences model to look at low-mortality death rates in hospitals before and after merging with an HMO. Further, we used logistic regression on California inpatient hospital data from OSHPD. The data set in total contained 47,442,140 patient discharges from 506 hospitals, with 151 measured variables from 2000-2011.

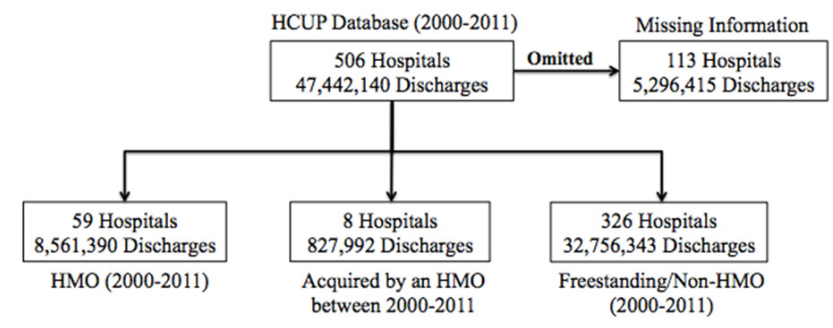

Figure 1. Methodological flowchart

Published by Sciedu Press
We sorted hospitals into three groups: hospitals that had merged with an HMO between 2000-2011, hospitals that had always been affiliated with an HMO, and hospitals that had never been associated with an HMO (control). Figure 1 depicts a representation of our selection process. We excluded 50 hospitals that reported zero discharges in a given year between 2000-2011.

First, we created a control group that consisted of hospitals that had sustained operations from 2000-2011. We studied the parent name of each hospital from 2000-2011 using utilization data provided by OSHPD, to identify hospitals that never had a parent name affiliated with an HMO. This group was made up of 328 hospitals and included 32,756,343 discharges.

Next, we sorted all hospitals that had sustained operations with an HMO from 2000-2011. To thoroughly classify this subset of hospitals, we identified all hospitals that maintained a given HMO parent name throughout this timeframe. This group consisted of 59 hospitals and 8,561,390 discharges.

Finally, we separated one group to include all hospitals that had vertically merged to an HMO between the years 20002011. To systematically determine which hospitals were merged with an HMO, we studied the parent name of each hospital from 2000-2011 to identify changes that had taken place. A change suggested that a hospital merged with another health care system. We further examined all hospitals that had a parent name change in this timeframe by reviewing insurance plan information from the Department of Managed Healthcare for the state of California to differentiate between HMO consolidations versus non-HMO integrated consolidations. This HMO-merged group consisted of eight hospitals and included 827,992 discharges.

The eight hospitals that were acquired by an HMO from 2000-2011 merged in different years. Figure 2 regresses freestanding and HMO-affiliated hospitals over time on lowmortality death rate (per 10,000). These data show a reduction in death rate in HMO but not freestanding hospitals. This observation suggested that we should control for variances in the healthcare system over time. To do so, we added a variable to assign a value corresponding to number of years pre/post merge to depict when the hospital had since merged (see Figure 3). For example, if a hospital merged in 2006, its 2008 data are assigned a 2 (years post-merge).

\subsection{Measuring quality of care}

The response variable we selected as a proxy for quality of care was the AHRQ Patient Safety Indicator \#2, death rate in low-mortality diagnosis related groups. We chose this measure because the underlying assumption of the Patient Safety 
Indicator is that when patients admitted for an extremely low-mortality condition or procedure die, a health care error across multiple components of the healthcare system is likely to be responsible. Thus, it attempts to assess coordination of care, which is what vertically integrated systems claim to improve. This Patient Safety Indicator describes in-hospital deaths per 1,000 discharges for low-mortality $(<0.5 \%)$ diagnosis related groups among patients ages 18 years and older or obstetric patients. In accordance with the Agency for Health Related Quality, the quality measure excludes cases with trauma, cancer, an immunocompromised state, and transfers to an acute-care facility. ${ }^{[16]}$ This indicator intends to identify in-hospital deaths in patients unlikely to die during hospitalization.

To study the health of the patient populations in both the experimental and control hospitals, we studied the prevalence of Type- 2 diabetes in each group using ICD-9-CM codes. We analyzed this variable to study the health of the patient populations in all respective hospital groups.

\subsection{Econometric models}

Each observation of our quality indicator is a binary variable, where $\mathrm{Y}$ is 1 if the patient died from a low-mortality condition. We used a differences-in-differences approach to study the differential effect of vertical integration with an HMO on quality. We used logistic regression analysis modeled by the equation shown below, where $\mathrm{X}$ is a vector of patient characteristics to study low mortality death rate in each respective hospital group (see Equation 1):

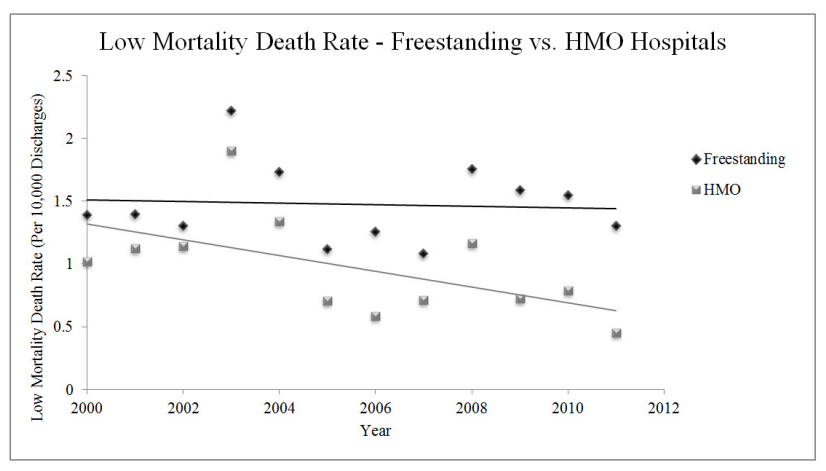

Figure 2. Regressing low-mortality death rates in freestanding hospitals versus HMO hospitals from 2000-2011 using simple linear regression

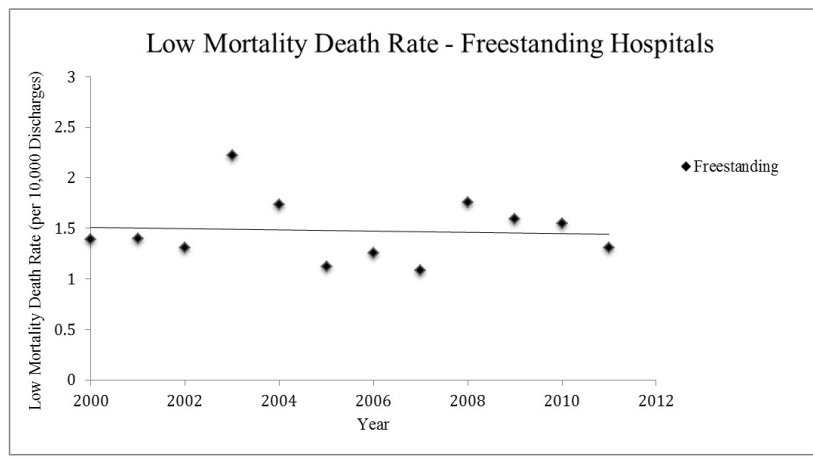

Figure 3. Regressing on low-mortality death rate for hospitals not affiliated with an HMO from 2000-2011 using simple linear regression

$$
\text { Low }- \text { mortality death rate }=\beta_{0}+\beta_{1} \text { Post }- \text { merge } \times H M O+\beta_{2} Y \text { ear }+\beta_{3} X+\varepsilon
$$

We conducted logistic regressions to control for demographic variables such as: age, race, gender, diabetes rate, and length of stay.

\subsection{Characteristics of the sample}

The characteristics of the different hospital groups that we focus on are important to understand. Table 1 reports summary statistics for the main control variables for the three categories of hospitals: freestanding hospitals, HMO hospitals, and HMO-acquired hospitals. Most hospital characteristics and patient demographics do not differ substantially across the different hospital types. The HMO hospitals experienced higher mean patient discharges per hospital than the freestanding hospitals from 2000-2011, and this same trend is followed by the HMO-acquired hospitals that increased mean patient discharges per hospital after merging. Further, the mean length of stay was higher in the freestanding hospitals than both the HMO and HMO-acquired patient populations. Shorter lengths of stay are typically associated with better 34 quality of care.

\section{RESULTS}

Our main finding best supports the notion that hospitals experience better coordination of care within a couple years of vertically integrating into an HMO. The AHRQ patient safety indicator "low-mortality death rate" is a measure of hospital quality. As such, we conducted our analysis regressing an interaction between hospital type and the post-merge time period on low-mortality death rate in Table 2, to identify which forms of administrative structures are linked to better care. Prior to being acquired by an HMO, these hospitals had higher low-mortality death rates than freestanding hospitals. However, the acquired HMO hospital-post-merge interaction term has a negative and statistically significant coefficient, suggesting that the hospitals experienced reductions in low-mortality death rates following a merge with an HMO. 
Table 1. Summary statistics for three groups of hospitals: freestanding, HMO-affiliated, and HMO-acquired hospitals from 2000-2011

\begin{tabular}{|c|c|c|c|c|}
\hline & \multirow{2}{*}{ Freestanding } & \multirow{2}{*}{ HMO } & \multicolumn{2}{|c|}{ HMO-Acquired } \\
\hline & & & Pre-Acquisition & Post-Acquisition \\
\hline Total Hospitals Included & 328 & 59 & 8 & 8 \\
\hline Total Patient Discharges & $32,756,343$ & $8,561,390$ & 429,523 & 398,469 \\
\hline Mean Patient Discharges/Hospital/Year & 8,322 & 12,092 & 8,516 & 9,738 \\
\hline Change in Patient Discharges (2000-2011) & $7.0 \%$ & $11.7 \%$ & & \\
\hline Mean Low Mortality Death Rate/10,000 Discharges & $1.47 \%$ & $0.97 \%$ & $1.94 \%$ & $0.66 \%$ \\
\hline Change in Low Mortality Death Rate (2000-2011) & $-5.7 \%$ & $-55.9 \%$ & & \\
\hline Mean Diabetes Rate & $14.3 \%$ & $15.5 \%$ & $16.6 \%$ & $22.4 \%$ \\
\hline Change in Diabetes Rate (2000-2011) & $72.0 \%$ & $65.3 \%$ & & \\
\hline Male & $32.8 \%$ & $31.9 \%$ & $33.5 \%$ & $33.7 \%$ \\
\hline Female & $48.9 \%$ & $49.7 \%$ & $53.3 \%$ & $53.7 \%$ \\
\hline White & $55.1 \%$ & $52.7 \%$ & $75.8 \%$ & $69.9 \%$ \\
\hline Black & $4.7 \%$ & $6.6 \%$ & $2.9 \%$ & $4.8 \%$ \\
\hline Asian & $4.8 \%$ & $4.8 \%$ & $1.4 \%$ & $2.4 \%$ \\
\hline Mean Age (years) & 42.7 & 44.5 & 53.4 & 53.1 \\
\hline Mean Length of Stay (days) & 5.4 & 4.1 & 4.4 & 4.1 \\
\hline
\end{tabular}

Table 2. Regressing on low-mortality death rate using a logistic model, with and without diabetes variable

\begin{tabular}{lll}
\hline & & (Excluding diabetes) \\
\hline HMO hospitals & $-0.4009^{* * *}$ & $-0.395^{* * *}$ \\
Acquired HMO hospitals & $0.2094^{* *}$ & $0.242^{* *}$ \\
Acquired HMO hospital-post-merge interaction & $-1.0097^{* * *}$ & $-1.000^{* * *}$ \\
Diabetes & $0.6429^{* * *}$ & -- \\
Length of Stay & $0.0013^{* * *}$ & $0.001^{* * *}$ \\
Year & $-0.0129^{* * *}$ & $-0.008^{* *}$ \\
Constant & $17.1621^{* *}$ & 7.79 \\
N & $35,611,548$ & $35,611,548$ \\
\hline
\end{tabular}

Note $^{* * * *}=$ Statistically significant at the .01 level; ${ }^{* *}=$ Statistically significant at the .05 level; ${ }^{*}=$ Statistically significant at the .10 level

According to Table 2, it appears that HMOs have improved low-mortality death rates compared to freestanding hospitals. Given the fact that total patient discharges are increasing faster in HMOs than in freestanding hospitals, patient selection may be responsible. Further, the negative coefficient on our time variable indicates that overall quality in all hospitals may be increasing over time. We obtained similar results for each variable with different model specifications, meaning that our estimates are robust. Omitting any control variable from our model does not change the results.

We regressed freestanding hospitals from 2000-2011 on lowmortality death rate to identify how quality changed over time. Figure 3 shows that the mean low-mortality death rate in freestanding hospitals was fairly consistent over time. In general, there was a slight decrease in low-mortality death rates from 2000-2011. This suggests that the hospitals in our analysis likely experienced rather modest increases in quality over time.
On the other hand, Figure 4 shows regression of hospitals over time, relative to the year they merged with an HMO, on low-mortality death rate. This demonstrates that, on the average, merging with an HMO was correlated with decreases in a hospital's overall low-mortality death rate. The data are broken out into two different time series groups, pre-merge and post-merge. Both groups had relatively the same, downward sloping trend. The greater magnitude of the pre-merge slope, in comparison to the slope of the freestanding hospitals from Figure 3, suggests that hospitals that were bought up in acquisition deals already had trends towards decreasing low-mortality death rates. However, merging with an HMO was associated with an overall reduction in the low-mortality death rate even after accounting for the trend, indicative from the decreased y-intercept value in the post-merge time series.

We regressed hospital groups and patient demographics on Type-2 diabetes rates to determine if HMO hospitals creamskimmed to obtain healthier patient populations. According 
to Table 3, the pool of hospitals that HMOs chose to acquire had higher rates of diabetes before the acquisition, and that rate increased a bit once the merge happened. The continued increase, coupled with the result that always-existing HMO hospitals experienced increased rates of diabetes as well, may be due to diabetes management programs attracting diabetes patients to the HMOs that offered them. This increase may suggest that HMO hospitals did not cream-skim the healthiest patients.

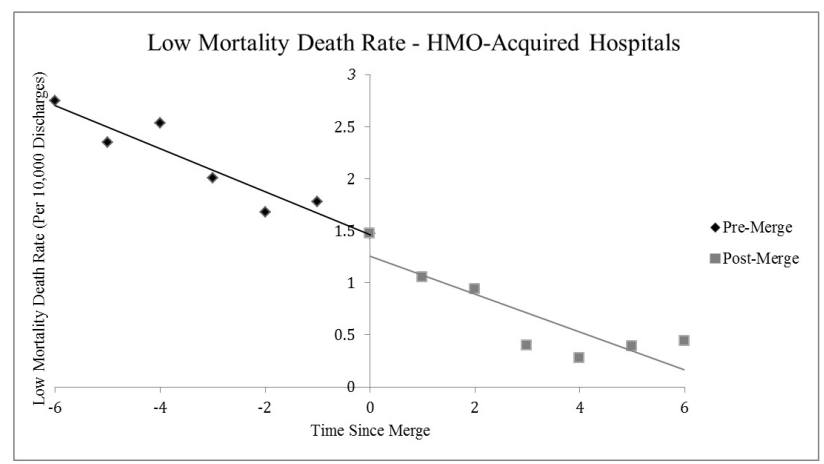

Figure 4. Regressing on low-mortality death rate for hospitals six years before and six years after a merge with an HMO using simple linear regression

Table 3. Regressing on diabetes rate using a logistic model

\begin{tabular}{ll}
\hline & Diabetes Rate \\
\hline HMO hospitals & $0.082^{* * * *}$ \\
Acquired HMO hospitals & $0.350^{\text {*** }}$ \\
Acquired HMO hospitals post-merge interaction & $0.082^{\text {**** }}$ \\
Female & $-0.279^{* * * *}$ \\
Black & $0.507^{\text {**** }}$ \\
Asian & $0.251^{\text {**** }}$ \\
Time & $0.054^{\text {**** }}$ \\
Constant & $-110.7^{* * * *}$ \\
N & $42,145,725$ \\
\hline Note. ${ }^{* * *}=$ Statistically significant at the .01 level &
\end{tabular}

\section{Discussion}

We analyzed the difference in quality of care delivered at hospitals before and after being acquired by an HMO. We found that hospitals experienced a drop in low-mortality death rates associated with the date of their merger. We do not believe this change is due to selection of patient populations because there was an increase in the diabetes rate in the post-merge hospital patient population consistent with the rising diabetes rates that freestanding hospitals experienced over time. Acquired hospitals had a greater proportion of white patients than that of the HMO and freestanding hospitals. However, all other races were associated with lower low-mortality death rates relative to whites. Thus, both findings suggest that the result of higher quality is not attributable to healthier patient populations, but perhaps rather to improved care following a merge with an HMO.

One possible explanation for increased quality in post-merge HMO-affiliated hospitals is that the new management may have instituted clinical protocol specific to the HMO to achieve system-wide standardization. In line with our findings, on the average HMOs have approximately 50 percent more discharges per hospital than freestanding hospitals. Comparably, hospital discharges increased 14 percent for hospitals per year following an HMO merge. In response to excess patient flow, HMOs likely used clinical protocol at a higher rate than other hospital types to maintain consistent "best practices" throughout its hospital system. Literature supports the effectiveness of protocol on positive patient outcomes on a wide range of different workflow processes and procedures. ${ }^{[17-20]}$ Standardized protocols decrease unwarranted variability, which could result in lower low-mortality death rates following a merge with an HMO, consistent with our findings. ${ }^{[18]}$

Further, it is possible that merging with an HMO allows a hospital access to a wider array of resources and more specialized care for their patients. HMO and post-merge patient populations were associated with shorter lengths of stay in comparison to freestanding and pre-merge hospitals. Patient access to specialized clinics due to a larger health network system in the HMO hospitals could be responsible for more focused and efficient care. Past findings have revealed that HMO-affiliated physicians and more specialized physicians were both more efficient, irrespective of patient illness characteristics. ${ }^{21]}$ Both results suggest that merging with an HMO could possibly lead to more specialized and efficient care, in turn increasing quality.

An alternative interpretation is that HMOs acquired higher performing hospitals that were trending towards increased quality, irrespective to the merge. This explanation is consistent with the steeper downward trend of the low-mortality death rate in the pre-merge hospital group. It therefore is possible that the acquired hospitals are not a representative sample to study the effects of an HMO merge on quality of care. However, we found that hospitals saw large reductions in low-mortality death rates the third year and beyond after merging with an HMO. These decreases are likely associated with the HMO, rather than the hospitals themselves. This finding is consistent with past literature that showed initial decreases in quality after converting to a for-profit hospital, but regained its initial quality performance the third year following conversion. ${ }^{[22]}$ Having made this distinction, the evidence may point to a switch rather than a continuation of a trend. 
Previous literature analyzing the relationship between managed care and quality show a relatively equal number of significantly better and worse HMO results for healthy patients, compared with non-HMO plans. ${ }^{[23,24]}$ Studies used multivariate regression in HMO versus non-HMO hospitals over time to study quality of care indicators, rather than pre- and post-HMO merge data like we did. Within differing HMO plans, the literature suggests that investor-owned HMOs deliver lower quality of care than not-for-profit plans. ${ }^{[25]}$ Nonethe-less, all of said research was conducted prior to the implementation of the Affordable Care Act, warranting further studies.

Prior research has examined the impact that hospital mergers have on quality of care. The majority of studies we reviewed found that increasing hospital market concentration has relatively no effect on quality. ${ }^{[26-28]}$ Of the studies reviewed, Carlin, Dowd and Feldman most closely resembled our study. ${ }^{[29]}$ They used administrative data for health plan enrollees in the clinics that were acquired by integrated delivery systems (IDS) in the Minneapolis-St. Paul area for two years prior to and four years after the acquisitions. They used a differences-in-differences model and estimated Probit models, similar to ours, with errors clustered within enrollees to compare changes in quality measures such as: breast cancer, colorectal cancer, and cervical cancer. The study found that the clinics that vertically integrated had slightly better quality outcomes. Thereby suggesting that IDS clinical acquisitions have the potential to improve patient outcomes, consistent with our findings.

Our study had several limitations due to the fact that we used patient outcome data. First, we are unable to know how accurate the patient outcome data are. The construct of the Patient Safety Indicator that we used relies on the assumption that every patient diagnosis was identified, correct, and documented by the hospital staff. Our study is dependent on hospital precision, and our merged hospital data set only contained eight distinct hospitals. As a result, poor documentation practices at one or more of these hospitals could possibly influence the validity of the outcome of this study.
Despite this drawback, the Patient Safety Indicators are still widely used in the literature. ${ }^{[30-32]}$

Second, the demographic data for each hospital group had missing values due to the fact that this statistic was selfreported. As a result, it is possible that gender and race proportions may have varied more than expected. This could have led to misinterpretations of the patient populations in our analysis.

\section{Conclusions}

We examined changes in the low-mortality death rate in three groups: non-HMOs, HMOs, and hospitals that vertically integrated into an HMO. We used a differences-in-differences model to study the effects on the OSHPD data set that included inpatient hospital data for patients in California from 2000-2011. Our findings suggest that merging with an HMO was associated with lower low-mortality death rates. We believe that merging with HMO hospitals could potentially result in better coordination in care, and increased quality of care as a result.

There are multiple ways in which this research can be meaningfully extended. First, there is a need to explore the prevalence of protocol in different hospital types. Evidence from that research can explain whether the decrease in lowmortality death rate observed from this analysis was simply due to increased use of protocol, or whether the cause is due to an alternative aspect of the HMO model.

Second, it is increasingly important to study those hospital characteristics that dictate the success of a merge in the healthcare sector at a time of frequent consolidations. Hospitals foreclosures result in hundreds unemployed and thousands seeking care. As previously noted, acquisitions are costly and risky thus it is important to understand aspects that contribute to the viability of hospitals following consolidation.

\section{CONFlicts OF InTEREST Disclosure}

The authors declare they have no conflict of interest.

\section{REFERENCES}

[1] American Hospital Association: trends affecting hospitals and health systems [Internet]. Trendwatch Chartbook. 2012 [23 January 2017, date last accessed]. Available from: http://www . aha.org/rese $\operatorname{arch} /$ reports/tw/chartbook/index.shtml

[2] The Henry J. Kaiser Family Foundation: total HMO enrollment [Internet]. [28 January 2017, date last accessed]. Available from: http://kff.org/other/state-indicator/total-hmo

Published by Sciedu Press -enrollment/

[3] Saxena S, Sharma A, Wong A. Succeeding in Hospital \& Health System M\&A. 2013.

[4] Aetna: 2014 Annual Report [Internet]. [21 January 2017, date last accessed]. Available from: https://materials.proxyvote.co m/Approved/00817Y/20150313/AR_239789/

[5] Anthem: 2014 Annual Report [Internet]. [21 January 2017, date last accessed]. Available from: http: 
//media.corporate-ir.net/media_files/IROL/13/13 0104/2014AR/export7/pdfs/Anthem_2014AR.pdf

[6] Humana: 2014 Annual Report [Internet]. [21 January 2017, date last accessed]. Available from: http://phx. corporate-ir.net/ph oenix.zhtml?c=92913\&p=irol-reportsannual

[7] Kaiser Permanente: 2014 Annual Report [Internet]. [21 January 2017, date last accessed]. Available from: https://share.kaiserperm anente.org/static/kp_annualreport_2014/

[8] UnitedHealth Group: 2014 Annual Report [Internet]. [21 January 2017, date last accessed]. Available from: http://www. unitedhe althgroup.com/2014-annual/Default .aspx

[9] Himmelstein DU, Jun M, Busse R, et al. A Comparison of Hospital Administrative Costs in Eight Nations: U.S. Costs Exceed All Others by Far. Health Affairs. 2014; 33(9): 1586-1594. https : //doi.org/10.1377/hlthaff.2013.1327

[10] Coase RH. The Nature of the Firm. Economica. 1937; 4(16): 386-405. https://doi.org/10.1111/j.1468-0335.1937.tb00002.x

[11] Williamson OE. The Economics of Organization: The Transaction Cost Approach. American Journal of Sociology. 1981; 87(3): 548577. https://doi.org/10.1086/227496

[12] Goodell S, Cohen J, Neumann P. Cost Savings and Cost-Effectiveness of Clinical Preventive Care. Princeton (NJ): Robert Wood Johnson Foundation; 2009 Sep. Report No.: 18.

[13] Maciosek M, Coffield A, Flottemesch T, et al. Greater Use of Preventive Services In U.S. Health Care Could Save Lives At Little Or No Cost. Health Affairs. 2010; 29(9): 1656-1660. https: //doi.org/10.1377/hlthaff.2008.0701

[14] Gaynor M, Vogt WB. Antitrust and competition in health care markets. Handbook of Health Economics. Volume 1, Part B: Elsevier; 2000. 1405-1487 p.

[15] Bendix J. MACRA Doesn't Spell End of Independent Medical Practices. Medical Economics. 2016.

[16] AHRQ: Patient Safety Tools and Resources [Internet]. [12 Dec 2017, date last accessed]. Available from: http://www.hcup-us.ahrq.gov/reports/statbriefs/ sb180-Hospitalizations-United-States-2012.pdf

[17] Hijazi M, Al-Ansari M. Protocol-Driven vs. Physician-Driven Electrolyte Replacement in Adult Critically Ill Patients. Ann Saudi Med. 2005; 25(2): 105-110. PMid: 15977686.

[18] Friedman SM, Mendelson DA, Kates SL, et al. Geriatric CoManagement of Proximal Femur Fractures: Total Quality Management and Protocol-Driven Care Result in Better Outcomes for a Frail Patient Population. J Am Geriatr Soc. 2008; 56(7): 1349-1356. https://doi.org/10.1111/j.1532-5415.2008.01770.x

[19] McCallie KR, Lee HC, Mayer O, et al. Improved outcomes with a standardized feeding protocol for very low birth weight infants. J Perinatol. 2011; 31(S1): S61-S67. https://doi.org/10.1038/ jp. 2010.185
[20] Ogilvie-Harris DJ, Botsford DJ, Hawker RW. Elderly Patients with Hip Fractures: Improved Outcome with the Use of Care Maps with High-Quality Medical and Nursing Protocols. Journal of Orthopaedic Trauma. 1993; 7(5): 428-437. PMid: 8229379. https: //doi.org/10.1097/00005131-199310000-00005

[21] Chilingerian J. Evaluating Physician Efficiency in Hospitals: A Multivariate Analysis of Best Practices. European Journal of Operational Research. 1995; 80(3): 548-574. https://doi.org/10.1016/03 77-2217 (94) 00137-2

[22] Picone G, Chou SY, Sloan F. Are For-Profit Hospital Conversions Harmful to Patients and to Medicare? RAND Journal of Economics. 2002; 33(3): 507-523. PMid: 12585305. https ://doi.org/10.2 $307 / 3087470$

[23] Miller RH, Luft HS. Does Managed Care Lead to Better or Worse Quality of Care. Health Affairs. 1997; 16(5): 7-25. https://doi. org/10.1377/hlthaff.16.5.7

[24] Hellinger FJ. The Effect of Managed Care on Quality: A Review of Recent Evidence. Arch Intern Med. 1998; 158(8): 833-884. PMid: 9570168. https://doi.org/10.1001/archinte.158.8.833

[25] Himmelstein D, Woolhandler S, Hellander I, et al. Quality of Care in Investor-Owned vs. Not-For-Profit HMOs. JAMA. 1999; 282(2): 159-163. https://doi.org/10.1001/jama.282.2.159

[26] Volpp K, Ketcham J, Epstein A, et al. The Effects of Price Competition and Reduced Subsides for Uncompensated Care on Hospital Mortality. Health Services Research. 2005; 40(4). PMid: 16033492. https://doi.org/10.1111/j.1475-6773.2005.00396.x

[27] Mutter R, Wong H. The Effects of Hospital Competition on Inpatient Quality of Care. Agency for Health Care Research and Quality. 2004.

[28] Shen YC. The Effect of Financial Pressure on the Quality of Care in Hospitals. Journal of Health Economics. 2003; 22(2). https: //doi.org/10.1016/S0167-6296(02)00124-8

[29] Carlin C, Dowd B, Feldman R. Changes in Quality of Health Care Delivery After Vertical Integration. Health Services Research. 2015; 50(4): 1043-1068. https ://doi.org/10.1111/1475-6773.12 274

[30] Cook A, Gaynor M, Stephens Jr M, et al. The effect of a hospital nurse staffing mandate on patient health outcomes: Evidence from California's minimum staffing regulation. Journal of Health Economics. 2012; 31(2): 340-348. https ://doi.org/10.1016/j.jh ealeco.2012.01.005

[31] Cuellar AE, Gertler PJ. Strategic integration of hospitals and physicians. Journal of Health Economics. 2006; 25(1): 1-28. https : //doi.org/10.1016/j.jhealeco.2005.04.009

[32] Rose J, Evans C, Barleben A, et al. Comparative Safety of Endovascular Aortic Aneurysm Repair Over Open Repair Using Patient Safety Indicators During Adoption. JAMA. 2014; 149(9): 926-932. https : //doi.org/10.1001/jamasurg. 2014.1018 